Published as: Vanclooster, A., Neutens, T., Fack, V., Van de Weghe, N. \& De Maeyer, P. (2012), Measuring the exitability of buildings: A new perspective on indoor accessibility. Applied Geography, vol. 34, pp. 507-518.

\title{
Measuring the exitability of buildings: a new perspective on indoor accessibility
}

\begin{abstract}
In the last decades, geographers' attention has been drawn to the vertical dimension of space and indoor environments due to population growth and concomitant city expansion. While traditional geography has long studied merely horizontal relationships of spatial processes and phenomena, recent years have also witnessed a growing number of studies that have sought to extend traditional spatial analysis tools to three-dimensional and indoor environments. In line with these developments, this paper proposes a new indoor accessibility measure which quantifies the quality of access to exits, called exitability. In this, the movement of people with respect to its three-dimensional environment, the user characteristics and the surrounding occupant interactions is considered key. Since the accessibility of exits is most important during evacuations, the calculation of exitability uses existing evacuation flow models. In a case study, we demonstrate the usefulness of exitability measurements through an application on existing building data.
\end{abstract}

\section{Keywords}

Accessibility, Indoor, Built environment, Pedestrian movement, Evacuation. 


\section{Introduction}

In the last decades, population growth and concomitant city expansion have exerted more and more pressure on urban space. Recent years have not only witnessed horizontal urbanized spreading, but also vertical building developments. These are triggered by a pinching deficit in land availability (e.g. Hong Kong), constructions of iconic single-phase mega-projects (e.g. Dubai) and enforced rules from governments revitalizing residential inner-city areas (Abel, 2010; Hwang, 2006; Wilson, 2010). The three-dimensional vertical city was born and with it the requirement of dealing with the corresponding complexities of multilevel building structures.

Past urban geographical research has unfolded the opportunities and limits of cities through extensive geospatial analysis (Ban \& Ahlqvist, 2009; Batisani \& Yarnal, 2009). Research of inner-city mobility (Antipova, et al., 2011; Keeling, 2008), accessibility analysis and studies of optimal time-space distributions (Kwan \& Weber, 2003; Neutens, et al., 2012; Neutens, et al., 2010; Versichele, et al., 2012) all reveal elements of the spatial distribution and interactions of people and businesses within the two-dimensional urban city.

In this paper, however, we focus on the city as a three-dimensional complex and more specifically on the multiple units that make up the 3D environment. We argue that spatial concepts need to be adapted to the intricacies of indoor environments, given the following differences between indoor and outdoor environments. First, the space itself is physically highly divergent. Outdoor space is considered mostly as non-built environment, not enclosed and large scale while indoor environments are mainly enclosed and constrained by the architectural infrastructure on a small scale (Li, 2008; Walton \& Worboys, 2009). Second, wayfinding tasks in multi-level buildings have proven to be more challenging than outdoors, for reasons of disorientation (due to multiple floor levels and staircases), and less visual aid (e.g. landmarks are less obviously recognizable; corners and narrow corridors prevent a complete overview) (Hölscher, et al., 2007). As such, building occupants are faced with a deficient perspective on the building structure, influencing their movement behavior (Hölscher, et al., 2007). Third, the scale level of analysis is for indoor building complexes more restricted than outdoors. Analysis techniques are required to cover the range of macroto microscale environments when combining indoor with outdoor space. As a result, the increased complexity of the three-dimensional vertical city induced by these differences can impact movement patterns and wayfinding choices of building occupants. Spatial analytical functions that focus on discovering and measuring this relationship between spaces and human movement will have to consider these intricacies.

With the increasing attention to the specificities of indoor spaces, the challenge was raised of adjusting analytical methodologies to the indoor environment. In this paper, we focus on one type of spatial analysis, namely accessibility. The aim is to examine accessibility within an indoor three-dimensional environment. A methodology will be put forward to analyze the accessibility of exits from building units (room-to-exit accessibility). Hence, the proposed accessibility measure will be termed exitability. The measure builds upon traditional outdoor accessibility concepts and extends those to the three-dimensional indoor environment. Relying on commonly used evacuation models, we will demonstrate how the concept of exitability can serve as a measure for the efficiency of the spatial building design in enabling evacuation of building occupants. 
The remainder of the paper is organized as follows. Section 2 elaborates on the definition of exitability and its relationship to accessibility. In section 3 , the model behind the exitability measure is discussed and framed within the existing state-of-the-art on evacuation modeling. In the case study in section 4, the exitability measure is calculated for a university building with multiple analyses showing its strength for spatial analysis of the 3D indoor environment. This paper is completed with a conclusion on the discussed issues.

\section{Exitability in relation to accessibility}

\subsection{Defining exitability}

To measure the quality of access to exit points, a function is required to objectively characterize spatial differences in access within and across buildings. For this, we develop a new type of indoor accessibility measure, termed exitability, which measures the occupants' ease of reaching exits within a building. Therefore, exitability is focused on the movement of the building occupants itself. This occupant movement depends on the structure of the spatial environment, including the topological building structure, the semantic structure and the building geometry, as well as the user environment, with the distribution of people per spatial unit. Access to exits is most important during emergency situations and the ensuing evacuation. As such, our definition of exitability accounts for movement of all building occupants to the exits during evacuations. For each room, it is calculated as the exit time needed for the movement of every occupant in the room to the exit. The total exitability of a building is quantified through averaging the individual exitability values of the separate rooms. The methodology for the calculation of exitability is explained in more detail in section 3.

\subsection{Analogies and differences with accessibility measures}

The developed exitability measure intersects with various threads of research. Its foundation relies on traditional outdoor accessibility measures. Both have a similar goal of quantifying the qualitative degree of connectivity between different places or persons (Kwan, 1999). Accessibility measures are widely used in urban transport and planning research as a tool to analyze and model activity patterns of customers in outdoor space (Kwan \& Weber, 2008; Neutens, et al., 2008). However, the setting for exitability has changed to the indoor threedimensional world. Exitability has also more strictly defined origin and destination sets. The interior building entities correspond to the origins while the exit features represent the destinations. In addition, the attraction of exit locations is modeled by closely considering the collective movement from building occupants to these exits.

Since exitability is defined for indoor environments, it is conceptually linked to indoor accessibility measures. So far, the latter has been developed from two divergent angles of research: (i) the quantification of individuals' indoor mobility limitations and (ii) spatial analysis of the built environment. The first strand of research aligns with a growing awareness of movement difficulties of people in buildings in the last decade (Sakkas \& Pérez, 2006). This has lead to requirements for building design and standards to measure and compare their proficiency at appropriately adapting space to everyone's needs. By considering buildings as user service providers, Sakkas and Pérez (2006), for example, defined indoor accessibility as a measure of quality of all representative service paths through a building. Church and Marston (2003), for their part, proposed a relative 
accessibility measure, which allowed the detection of access differences relative to distinct user groups. Beside these theoretical approaches, the European Union developed the European Passe-Partout index ("Le Site Officiel de l'Indice Passe-Partout (IPP)," 2012) as a method to assess the accessibility of buildings with regard to disabled people, following legal recommendations from various countries. This index lists for every building how well it is adapted to the specific requirements of persons with limited mobility based on predefined parameters. These indoor accessibility indices are mostly used as recommendations for adapting existing buildings to the requirements of physically impaired persons (Otmani, et al., 2009), limiting their scope to solely this specific group of people. However, when assessing the general accessibility of building exits, all building occupants should be taken into account, while still retaining a high interest in previously considered groups. Therefore, these indoor accessibility measures cannot be used as a model for grasping the spatial interrelationships between multiple building units.

The second line of inquiry includes recent work from Kim et al. (2008) and Thill et al. (2011) which demonstrates the calculation of accessibility measures in buildings by considering human movement. They both use a different methodology, with Kim et al. (2008) buttressing up their method with the space syntax theory; while Thill et al. (2011) employ a traditional gravity-based model. Apart from their incorporation in a three-dimensional built environment, both approaches calculate the accessibility of a single spatial unit with regard to pedestrian movement under non-emergency situations, while in our research, exitability is measured under evacuation scenarios. Also, our calculation is based on the actual movement of occupants and not like the aforementioned approaches based solely on distance and geometric characteristics of the building. With these limitations, none of the currently available indoor accessibility measures is able to fully quantify the quality of access to exits during evacuations, on which we focus in this paper.

\section{Methodology for calculating exitability}

\subsection{State-of-the-art in evacuation modeling}

Evacuation analysis and response has a wide interest for various researchers in understanding and preventing hazardous situations (VanLandegen \& Chen, 2012). Partly due to a string of major world events (e.g. attacks on the WTC in 2001, London bombing and hurricane Katrina in 2005), the need for developing evacuation models for building environments has grown progressively over the last decades. This renewed interest brought along a boost in the development of sophisticated computer simulation models.

Historically, studies on building evacuation modeling originated from pedestrian movement models since the 1970s. In these studies, human behavior and movement was quantified and modeled under both non-emergency and emergency conditions and this mostly from a static context (Cepolina, 2004; Gwynne, et al., 1999). From this period onward, flow-based mathematical formulas became widely available (e.g. the formulas from Fruin (1971) and Predtechenskii and Milinskii (1978)) (Hamacher \& Tjandra, 2001; Santos \& Aguirre, 2004). A second research surge began in the early 1980s with the development of computer simulations for evacuation modeling (Gwynne, et al., 1999; Hamacher \& Tjandra, 2001; Santos \& Aguirre, 2004). Here, at least two strands of research can be recognized. First, ball-bearing, fluid-dynamic and flow-based models extended the mathematical flow models with individual occupant modeling and queuing. However, these aggregate models still 
treated individuals as homogenous groups acting together (Castle \& Longley, 2008; Santos \& Aguirre, 2004) with the speed and direction of human movement determined by physical constraints. The aforementioned models were later on slowly replaced by individual level modeling with humans as active agents, which made it possible to link human movement with human behavior (Gwynne, et al., 1999). The development of automata allowed for the processing of dynamic characteristics (Castle \& Longley, 2008; Gwynne, et al., 1999).

Based on the above review, we can draw some conclusions on the existing models and the remaining research challenges. First, a multitude of highly complex and sophisticated simulation models is available for evacuation and pedestrian movement. The chosen model for a certain application depends on the purpose of the application, the scope and the requirements on among other things the level of detail, input data, output, computational strength and runtime (Kuligowski, 2008). Second, many parameters influence the evacuation process, ranging from the characteristics of the emergency situation to the human reaction and behavior, user experience and built environment. Even within this research field, there is no consensus yet on the correct implementation of all these parameters; with criticism especially towards the method and data of human behavior incorporation (Averill, 2010). Gwynne et al. (1999) recognized a trend towards implementing more and more behavioral characteristics to match the real human reaction in case of emergencies. More recently, Zheng et al. (2009) confirmed this trend by proposing combinations of various approaches to study crowd evacuation, employing rules from one approach on the basic principles of the other approach. However, no evacuation model already fully addresses all behavioral aspects involved in emergency situations and evacuations. Additionally, not all of the behaviors involved are yet fully understood and analyzed (Gwynne, et al., 1999; Kuligowski, 2008).

With the above conclusions in mind, we chose to employ a coarse network flow model from a global perspective with homogenous mapping of occupants and queuing. While this model is used for calculating the exitability based on flow movement during evacuations, it is important to emphasize that the evacuation principle is not the main parameter of interest here, but is solely comprehended as the most stringent situation precluding optimal accessibility. The focus is on the general level of exitability within buildings, not on the effects of a particular emergency event on occupant movement (as is generally the case in previous work). Therefore, the individual and random characteristics of an emergency situation and ensuing evacuation itself are left unconsidered. This allows us to make not only comments about the accessibility during evacuations, but also under non-emergency situations and their effects on particular spatial inter-room differences.

\subsection{Calculation of exitability}

\section{Spatial model}

For calculating exitability, a representation model of the enclosure space is required, in this case a three-dimensional data model that represents the internal structures of the built environment. We employ a coarse network model implemented as a network graph that discretizes space into subregions, all internally connected (Gwynne, et al., 1999). This has the advantage of representing all necessary topological relationships between the spatial building units while preserving a close connection with the actual movement of human beings (Lee, 2004). The model is equivalent to the widely used 'Geometric Network Model' (GNM) of 
Lee (2004) where the pure connectivity graph ('Combinatorial Network') (Figure 1a), containing solely topological relationships, is transformed into a geometric network (Figure 1c). This is attained through enhancing the 'Combinatorial Network' with geometry information and creating a subgraph for linear phenomena (e.g. corridors) into the node-edge structure.

$<$ Insert Figure 1>

As such, the GNM is an abstraction of every building's connectivity structure with additional geometric information enabling network analysis equivalent to road network analysis. Additional information necessary for this analysis can be stored in either the nodes or the links interconnecting these nodes, or in both.

\section{Flow model}

For calculating exitability, the occupants' movement is represented as a continuous flow of homogenous groups of people (Santos \& Aguirre, 2004). Flow-based evacuation models are commonly based on the following assumptions (Kratchman, 2006):

(1) All persons will start to evacuate at the same time;

(2) Occupant flow will not involve any interruptions caused by decisions of other building users;

(3) All or most of the persons involved are free from disabilities that would significantly impede their ability to keep up with the movement of a group.

The above mentioned assumptions will also apply to the calculation methodology of exitability for different reasons. For example, the first assumption implies that pre-movement times are omitted in the calculation of the final evacuation times. The pre-movement time in evacuations is the time for occupants to detect and respond to the emergency situation (Fahy \& Proulx, 2001; Gwynne, et al., 2003). A multitude of data on delay times has already been collected from various studies, but using them should be done with the highest prudence (Fahy \& Proulx, 2001; Gwynne, et al., 2003). After all, mistakes are frequently induced in the sense that the original context of the data is often lost and ignored (Gwynne, et al., 2003) and the data is mostly building, situation and occupant specific (Fahy \& Proulx, 2001). Also, an evacuation model with no or less behavioral perspective and homogenous groups (like the one applied in our calculation) might benefit from not implementing these delay times given the inherent focus on group behavior rather than individualism. After all, pre-movement times are a simplification of the behavioral process due to an emphasis on the time delay rather than on the decisions and actions of occupants responding to the evacuation itself (Kuligowski, 2008). The second assumption implies that occupants are homogenously modeled without any personal decision making and behavior. People will continuously keep moving in the direction of their choice, only hindered by co-occupants on the same path influencing the flow density. In current evacuation modeling research, a dichotomy exists between behavioral (individualistic) and non-behavioral (group) modeling of occupants (Gwynne, et al., 1999; Kuligowski, 2008). This assumption and our calculation is in accordance with the homogenous group modeling. As discussed in section 3.1, there is no consensus yet on a comprehensive methodology for modeling human behavior, with current models using significant simplifications of the behavioral processes during evacuations (Kuligowski, 2008). Their implemented behavior is either predefined by the user or based on inconsistent prescribed information entirely dependent on the user's expertise (Kuligowski, 2008). Also, behavioral modeling would significantly increase the complexity and 
computational requirements, and differentiations between randomly imposed behavior are not crucial to grasp differences in quality of access of exits. For these reasons, we opted to leave behavioral decision patterns out of the calculation methodology and only focus on the actual movement of the occupants influenced by density variations due to co-occupants' movement. The third assumption recalls the focus of the model to non-disabled persons making it more general than some of the current indoor accessibility measures only focusing on disabilities (Section 2).

\section{Network flow calculations}

The calculation of exitability is defined by the flow of building occupants departing from the central node in each room. Their movement speed is determined by the group density, which can change over time, and by the maximum capacity constraints of each edge, which in turn are determined by the minimum width of the passageways. This minimum width is used as approximation of the maximum possible walking space since in reality groups of people spread out to the maximum available space (Yuan, et al., 2009). The crowd density varies with time and location according to the non-uniform distribution of occupants. The formulas to calculate the speed are based on the pedestrian flow model of Predtechenskii and Milinskii (1978). Since the movement speed of people not appears to have changed over the years, this flow model can be and is still widely applied in other models (Fahy \& Proulx, 2001).

The crowd density $(D)$ of a stream of people is calculated in this model as a fraction of the number of people $(M)$ and the personal space area $(f)$ on the occupied space (Figure 2 and equation 1). The personal space area is the area in which no other person will move. It is based on the mean dimensions of an adult in mid-season street dress (Fahy, 1994) and has a fixed value of $0.113 \mathrm{~m}^{2}$. The stream is calculated for a certain occupied area, limited by the maximum width of personal interaction $(\delta y)$ and the maximum length of possible interaction for a person $(\delta x)$. The interaction width can be taken approximately as the maximum width without obstacles of the spatial unit. The length of occupant interaction is set as $1 \mathrm{~m}$ and records as such the number of people moving in the $1 \mathrm{~m}$ area around the occupant.

$$
D=N f / \delta x \delta y\left(\mathrm{~m}^{2} / \mathrm{m}^{2}\right)
$$

<Insert Figure 2>

In non-emergency situations, the mean velocity in open horizontal space ( $V$ can be calculated, using equation 2 , as a function of the crowd density based on observations of people walking (Predtechenskii \& Milinskii, 1978):

$$
V=112 D^{4}-380 D^{3}+434 D^{2}-217 D+57(\mathrm{~m} / \mathrm{min}) \text { for } 0<D \leq 0.92
$$

The crowd density has an optimal value of $0.92 \mathrm{~m}^{2} / \mathrm{m}^{2}$, although higher values are accepted. However, empirically this is used as the maximum allowed density (Fahy, 1994). In emergencies, the movement speeds $\left(V_{e}\right)$ are somewhat different with the same densities, since people are reacting more anxiously. Equation 3 shows the relationship between these two velocities, differentiating between movement through openings and in horizontal space, and movement on stairs (Predtechenskii \& Milinskii, 1978):

$$
V_{e}=\mu_{e} V \quad(\mathrm{~m} / \mathrm{min})
$$




$$
\text { where } \begin{aligned}
\mu_{e} & =1.49-0.36 D \\
\mu_{e} & =1.21
\end{aligned}
$$

(for horizontal paths and through openings)

(for descending stairs)

Queuing is handled by combining different groups when they meet each other, reducing their velocity and adding waiting times. As a result the maximum capacity on each edge may be reached.

\section{General workflow of the model}

In our flow-based movement model, occupants move from a room to the closest exit - that is along Dijkstra's (1959) shortest path (distance-based). However, it would also be possible to use the most familiar route or the shortest time to the exit, but this implementation is left for future work. The model does not allow dynamic changes in exit choice, which implies that all occupants follow the physically shortest path leaving personal decision making unconsidered.

The main parameters in the flow calculation are Path, NodeMovement and PassingNodeMovement objects ${ }^{1}$. Per room (and thus source node) a Path object is created storing the shortest path to the selected exit for this room. A NodeMovement object represents a group of people moving along an edge from start node to end node. This makes it easy to obtain the current position of each group (per time and location) during the evacuation. It also allows modeling the flow of people within a certain passing node over time. Every passing node stores a PassingNodeMovement object containing lists of NodeMovements with the arrival times and waiting times for every source node passing through this passing node.

The main idea behind the flow model is the merging and moving of the crowd to their closest exit taking into account in- and outflows of adjacent nodes. The main method Algorithm loops through all paths starting with the path with the shortest distance to the selected exit. With every selected path, the method EdgePassing runs over the entire path from its source node to the selected exit. In this loop, every subsequent edge between two nodes is selected, starting with the source node and ending when the exit node is reached. Within this method, flows are checked for incoming and outgoing groups to and from the start node of the edge. Then, the population is moved over the selected edge from start node to end node.

The incoming flows of groups of people coming from adjacent nodes are continuously calculated in every passing node using the IncomingFlows method (Figures 3 and 4). This method checks for every edge arriving in the passing node whether groups of people can possibly interact with the currently selected arriving group. Only groups arriving before or together with the selected group can interrupt its movement (line 7). Groups arriving earlier in the selected passing node have no direct impact on the selected NodeMovement in incoming times. However, they can still have a delaying effect on the outflow of the selected NodeMovement. The program checks then recursively (line 8) for subsequent NodeMovements along the same path, until the resulting time frames overlap. Overlap is treated through attaching waiting times or merging both groups, depending on the relationship between both timeframes (lines 12-18). The procedure stops when all possible interacting flows are calculated in the selected passing node.

\footnotetext{
${ }^{1}$ The different object classes are indicated with a capital letter and in italic. The methods are in bold.
} 
$<$ Insert Figure 3>

$<$ Insert Figure 4>

The outgoing flow from this selected passing node can be interrupted by preceding NodeMovements moving at a slower speed, which can result in catching up and overtaking of groups (Figures 4 and 5). All NodeMovements will form a queue of consecutive groups moving at the speed of the first group. The outgoingFlow procedure calculates this by iterating over all NodeMovements arriving in the selected passing node until the originally selected NodeMovement is reached. If an overtaking risk exists within the movement over the selected edge, the speed is adapted to that of the predecessor (line 8). This group is then selected and the method EdgePassing is invoked moving this group further towards the exit. Afterwards, the outgoingFlow method will pick up from the originally selected NodeMovement moving the group to the next passing node.

$<$ Insert Figure 5>

At the end of the outgoingFlow method, the selected group will be assigned a certain evacuation time. The whole process starts over again by selecting the next Path object in the method Algorithm until all paths are scanned and the different evacuation times are known.

\section{Case study}

The goal of this case study is to show the capabilities of exitability for spatial analysis of indoor environments and its added value of interpreting inter-room differences in exitability. Questions to be answered include (but are not restricted to): How accessible is a certain exit?; What is the least accessible area in the building?; How does the exitability change with changing population?; and How many people can exit the building within 5, 10 or 15 min?.

For this analysis, an existing building (S9) on the University Campus De Sterre in Ghent (Belgium) was used. This four-story building has three main exits and one evacuation exit. The main exits are situated on opposite sides of the longest side of the building with two exits closely connected (Exit 2 and 3 ). The building consists of four main lecture halls, three computer rooms, two smaller lecture rooms and many offices. These different compartment types correspond to a varying population density. Staircases, exits and corridors have no population since they are mainly used as connectors for movement between the various compartments. Rooms are one to seven person offices while the lecture halls can accommodate between 50 and 300 people each. The total maximum population of the building is 1446 occupants. Figure 6 visualizes the spatial location of the various compartments with their corresponding population. For this case study, the building was digitized and transformed into a dataset of nodes with id, room number, room type and population; and edges, with id, start node, end node, cost of the edge, minimal passage width and type of the link. The dataset consists of 213 nodes and 470 unidirectional edges.

<Insert Figure 6>

We will discuss two separate issues: a basic scenario with various questions with regard to the buildings exitability and secondly some scenarios where we change the original dataset to see how changes in environmental parameters affect the exitability of the building. 


\subsection{Spatial analysis of the base scenario}

In this scenario, the building is completely occupied with every compartment having its maximum number of building occupants. All four exits are available for evacuation with the exit choice for the single building occupant based on the shortest distance of the relevant room to the closest exit node. Table 1 shows the population load of each exit. Exit 2 and 3 are joined since no differences in exitability can be detected between both exits due to their opposite location. From this table, it can be concluded that overall a major discrepancy exists in the load of the three exits as exit 1 handles the majority of the total building occupants (more than $50 \%$ ).

$<$ Insert Table 1>

How exitable is this building? What are the least accessible areas? Figure 7 shows the result of the exitability calculations for the building with the individual exitability values per room. The spatial distribution of arriving times shows that the best exitability can be found in the rooms adjacent to the exits and the stairs, while more distant rooms have much higher values. The highest exitability values are found on the top floor and in the main lecture halls. These areas prove to be the most vulnerable in case of evacuations and require special attention. Some rooms have considerably higher exitability values than their neighboring rooms, due to higher population rates and queuing. For example, the offices in the main corridor on floor 1 have a similar population and distance to the exit but some rooms show worse exitability values due to congestion. The total maximum exitability is 626 seconds for the main lecture hall on the first floor. The average exitability is fairly low with 180 seconds with a standard deviation of 147 . Figure 8 shows the percentage of people who are able to leave within a range of 1 to 10 minutes. It is demonstrated that 50 percent of the building occupants can reach the exit within 5-6 minutes and $95 \%$ of the building can be evacuated within 10 minutes.

$<$ Insert Figure 7> $<$ Insert Figure 8>

What is the influence of distance on exitability? Previous results showed that higher floors have higher exitability values. This proves to be a logical result due to the direct relationship between the physical closeness of those rooms to their selected exit and the times needed for evacuating. Figure 9 supports this claim with an almost linear relationship between distance and exit times for some source nodes, clearly subdivided per floor (solid ellipses). Rooms on higher floors have considerably higher exitability values given the flocking effect near stairs along the path to the exit. In fact, those stairs can be seen as intermediate exit points and the effect of walking towards stairs is similar as the effect of walking to an exit. However, several outliers create a more nuanced view. Higher floor levels show more variability in values per level (e.g. more differences in colors in figure 7). This supports the spatial pattern of exitability values with fast evacuations for rooms close to the stairs and slower exitability for rooms in the middle of the central corridors (delayed by slower groups and main lecture halls). Also, the dashed ellipses in figure 9 group source nodes with high population densities (e.g. the main lecture rooms on the south end of the building), showing higher exitability values than expected due to a slower movement of each group. This slackened movement also has a delaying effect on subsequent groups of people from adjacent source nodes. The dotted ellipses show these rooms which tend to be located in the 
middle of the central corridors and are hindered by movement of the rooms closer to the stairs. They have higher exitability values than expected given their population and location. In contrast, some rooms have low exitability values even with long shortest paths. This positive influence is caused by low population values and unhindered movement to the exit given their immediate closeness to the stairs (no congestion due to predecessors).

$<$ Insert Figure 9>

How accessible are the exits? The distribution of the exitability values differs with the exit choice (Figure 10). Most rooms are closest located to exit 1, resulting in on average rather low exitability values. This means that rooms evacuating through exit 1 are able to get out in a fairly fast way, even with a heavier load on this exit. The statistical values for exit 2 show a reasonably concentrated distribution with slightly higher exitability values. Only four rooms (i.e. main lecture rooms) use exit 4 in case of an evacuation, resulting in less congestion even with the high occupancy rate. The average exitability rate for the entire building is 330 seconds. Occupants exiting through exit 1 and 2 have $5-10 \%$ higher averages, while the average exitability for exit 4 is $20 \%$ lower than the average for the building. This lower value is influenced by the reduced number of compartments evacuating through this exit and a smoother occupant movement. Movement to exit 2 is the most unfavorable given the fact that a reduced occupant load on this exit results in higher exitability values.

$<$ Insert Figure 10>

How does the exitability change with only 1 exit available? In emergency situations, some exits might be unavailable for evacuations. This spatial concentration of exit possibilities leads in the extreme case to only one usable exit which in turn results in a drastic decrease in available exit routes. Since the data set contains three building exits, this scenario is subdivided in three cases, one for each exit. Figure 11 shows the statistical distribution in each case and for comparison reasons also the distribution of the base scenario with all exits in use. Figures 12, 13 and 14 show the exitability results per available exit. From these visualizations, it can be concluded that a decline in available exit possibilities with the same spatial population distribution has a major influence on the resulting exitability values.

$<$ Insert Figure 11>

The results show that evacuations along exit 1 run quite smoothly. This is similar to the base scenario where already many occupants usually use this exit. As a result, the extra load on this exit (i.e. from occupants normally using exits 2 and 4 ) has no significant effect on the total exitability of the building. Additionally, exit 1 has the largest opening width which accelerates the evacuation process even more. However, the exitability values are in comparison with the base scenario on average higher and with a greater internal distribution (Figure 11). A similar view can be detected for evacuations along exit 4, although the effect is worsened. Occupants from the main lecture halls still have immediate access to the exit (due to its physical closeness), but a slackening effect occurs to the groups following. This is a result of the slower processing of the large groups from the lecture halls and the considerable smaller door width of the exit. This in turn affects the exitability values of rooms further removed from the exit queuing behind the preceding slower groups. The scenario with only exit 2 available is the most alarming for lecture halls opposite to the exit. Occupants from those rooms have to walk considerably further and are impeded on their way to the exit 
by predecessors and smaller opening and corridor widths. The distribution of the different values are however similar to the other scenarios with higher base values (Figure 11).

Secondly, figures 12,13 and 14 show that the exitability values differ over the multiple floor levels, with the lowest value on the ground floor and the highest values on the top floor. This is consistent with the direct relationship between distance and evacuation time. However, the data show a striking phenomenon with the more unfavorable exitability values from floor level 1 compared to those from level 2. This is attributed to the initial congestion originating from occupants from level 1, while occupants on level 2 have to traverse a longer distance and at the time arriving on level 1 already have to deal with less congestion and hinder from predecessors.

Thirdly, rooms with an average occupancy rate and an immediate connection to the stairs hold higher exitability values than rooms further away. However, the occupancy rate may result in a deteriorating effect (e.g. the main lecture rooms in the south).

$<$ Insert Figure 12>

$<$ Insert Figure 13>

$<$ Insert Figure 14>

\subsection{Effect of population and corridor width on exitability}

How does the population distribution influence exitability? The capability of the building is tested for coping with a drastic population decrease which corresponds to reality since during holidays the lecture and computer rooms are not used. Compared to the base scenario the whole population is more than 5 times smaller with only occupants in the offices resulting in a total of 248 persons.

Figure 15 shows that the exitability values decrease with decreasing occupancy. All rooms have considerably lower exitability values, with inter-room differences attributed to disparities in physical distance and the slight difference in occupancy rate for some rooms. The result also shows a more linear relationship between distance and exitability values compared to the base scenario (although slower movement on stairs and discrepancies in occupancy rates impedes perfect linearity).

$<$ Insert Figure 15>

How does a decreased corridor width change exitability? The corridor width of the main corridors on the different floor levels was narrowed from $4 \mathrm{~m}$ to $2 \mathrm{~m}$ to be more realistic with the presence of cupboards preventing the complete use of the corridor. This test allows examining the influence of the physical building characteristics on exitability. Figure 16 shows that the effect of smaller corridor widths is minimal on the exitability values in this case study. This can be explained by the limited number of occupants that is affected by this change in corridor width along their path to the exit. As shown previously, the main lecture halls with high occupancy rates can considerably deteriorate the evacuation process. However, half of the building occupants in this scenario have the same evacuation path characteristics as in the original context. In this case, only some rooms are affected with a slightly higher exitability and this mainly on floors 2 and 3 . After all, they have to travel the longest path and are more sensitive to congestion and queuing behind slower predecessors. 
The other trends described above are similar for this scenario with major distance influence and primarily congestion from highly populated rooms.

$<$ Insert Figure 16>

\section{Conclusion}

In this paper, we have put forward a new indoor accessibility measure, termed exitability, to analyze the accessibility of exits from within the various spatial building units (room-to-exit accessibility). Since exitability portrays the easiness with which occupants can reach building exits, it focuses on the movement of the building occupants from their internal building location to the exit. The calculation methodology is based on flow models and is illustrated in a case study regarding the efficiency of a spatial building design and room occupancy on the ease to evacuate a building. The results obtained in the case study indicate the importance of the physical distance on exitability. The further physically removed from an exit, the higher the chances that the exitability will be worse compared to rooms nearby. This effect is however modulated by the flow size of building occupants. In particular, congestion or extended population movement results in higher exitability values than expected on grounds of spatial proximity alone.

For the building considered in the case study, no significant problems were detected with regard to the quality of access of the various rooms (e.g. all rooms have within 10min access to an exit). While the results of course specifically apply to this particular building with a certain population distribution and building context, it is important to highlight the more general advantages and possibilities with calculating exitability. First, comparing room values of exitability can result in showing major discrepancies between rooms or floor levels which show the quality of the building design. For example, it allows one to see how changes in parameters like corridor or door width or the position of exits might affect the overall exitability of a building and show the need of changing design configurations. Also, the accepted population distribution can be analyzed with regard to the exit load or the spread per floor level, which can result in changes to allow a more optimal exitability. In addition, clusters of rooms with worse exitability can be detected which might be not noticeable at first sight. In addition, several buildings can be compared in terms of overall exitability to reveal which buildings allow to be cleared more easily.

The contribution of our work to the academic literature is at least two-fold. First, with respect to evacuation modeling, we have demonstrated the possibilities of spatially analyzing a building's feasibility of dealing with emergency situations. Second, exitability quantifies a qualitative relationship of access. As such, it can be used to optimize space-time decisions for users within buildings. The extension towards indoor environments is in line with the gradual refocus of geospatial applications towards the three-dimensional indoor built environment. Exitability also deals with the constraints of indoor environments. Previous indoor accessibility measures have been developed either for pointing out mobility issues for the physically impaired or for spatial analysis. Our work fits in with the latter, but tries to calculate accessibility not based on solely geometrical parameters, but also on actual movements of people.

As future work, an extension to this exitability measure can be considered, where exitability is calculated under non-emergency situations and even with different destination points. In 
that case, exitability is closer defined to the traditional accessibility measures. Adaptation to this concept opens the world to analysis of accessibility in all situations. As such, we believe that we made valuable contributions with our research to a better understanding of the intricacies of indoor environments.

\section{Captions}

Figure 1: Design of the 'Geometric Network Model' (Source: Lee (2004)).

Figure 2: Parameters in the pedestrian flow model.

Figure 3: Algorithm of the IncomingFlows in a selected passing node.

Figure 4: Movement in a passing node.

Figure 5: Algorithm of the OutgoingFlows in a selected passing node.

Figure 6: Population distribution in the base scenario.

Figure 7: Exitability values for evacuation towards all exits in the base scenario.

Figure 8: Ratio of people able to exit within a certain time limit.

Figure 9: Comparison between shortest path and exitability.

Figure 10: Distribution of exitability over the various exits in the base scenario.

Figure 11: Distribution of exitability over the various exits with only 1 exit available.

Figure 12: Exitability values for evacuation towards exit 1.

Figure 13: Exitability values for evacuation towards exit 2/3.

Figure 14: Exitability values for evacuation towards exit 4.

Figure 15: Exitability values for evacuation towards all exits with decreased population.

Figure 16: Exitability values for evacuation towards all exits with decreased corridor width.

Table 1: Distribution of the population over the different exits.

\section{Acknowledgements}

Financial support from the Research Foundation Flanders (FWO) is gratefully acknowledged. The use of the building CAD files was kindly permitted by the Department of Infrastructure and Facility Management of Ghent University.

\section{References}

Abel, C. (2010). The vertical garden city: towards a new urban topology. CTBUH Journal (2), 20-30.

Antipova, A., Wang, F., \& Wilmot, C. (2011). Urban land uses, socio-demographic attributes and commuting: A multilevel modeling approach. Applied Geography, 31 (3), 10101018.

Averill, J. D. (2010). Five Grand Challenges in Pedestrian and Evacuation Dynamics. In 5th International Conference on Pedestrian and Evacuation Dynamics (pp. 3-4). NIST, Gaithersburg, MD.

Ban, H. W., \& Ahlqvist, O. (2009). Representing and negotiating uncertain geospatial concepts - Where are the exurban areas? Computers, Environment and Urban Systems, 33 (4), 233-246.

Batisani, N., \& Yarnal, B. (2009). Urban expansion in Centre County, Pennsylvania: Spatial dynamics and landscape transformations. Applied Geography, 29 (2), 235-249.

Castle, C. J. E., \& Longley, P. A. (2008). Building Evacuation in Emergencies: A Review and Interpretation of Software for Simulating Pedestrian Egress. In D. Z. Sui (Ed.), Geospatial Technologies and Homeland Security (Vol. 94, pp. 209-228). Springer. 
Cepolina, E. M. (2004). Building Evacuation: Two Different Approaches. In European Transport Conference 2004 (pp. 12), Strasbourg, France.

Church, R. L., \& Marston, J. R. (2003). Measuring Accessibility for People with a Disability. Geographical Analysis, 35 (1), 83-96.

Dijkstra, E. W. (1959). A Note on Two Problems in Connexion with Graphs. Numerische Mathematik, 1 (1), 269-271.

Fahy, R. F. (1994). Exit 89 - An Evacuation Model for High-Rise Buildings - Model Description and Example Applications. In Fourth International Symposium of Fire Safety Science (pp. 657-668), Ottawa, Canada.

Fahy, R. F., \& Proulx, G. (2001). Toward creating a database on delay times to start evacuation and walking speeds for use in evacuation modeling. In 2nd International Symposium on Human Behaviour in Fire (pp. 175-183), Boston, MA., U.S.A.

Fruin, J. J. (1971). Pedestrian planning and design. Metropolitan Association of Urban Designers and Environmental Planners, New York.

Gwynne, S., Galea, E. R., Owen, M., Lawrence, P. J., \& Filippidis, L. (1999). A Review of the Methodologies Used in Evacuation Modelling. Fire and Materials (23), 383-388.

Gwynne, S., Galea, E. R., Parke, J., \& Hickson, J. (2003). The Collection and Analysis of Pre-evacuation Times Derived from Evacuation Trials and Their Application to Evacuation Modelling. Fire Technology, 39 (2), 173-195.

Hamacher, H. W., \& Tjandra, S. A. (2001). Mathematical Modelling of Evacuation Problems: A State of Art. In. Institut Techno- und Wirtschaftsmathematik.

Hölscher, C., Büchner, S. J., Meilinger, T., \& Strube, G. (2007). Map use and wayfinding strategies in a multi-building ensemble. In 2006 international conference on Spatial Cognition V: reasoning, action, interaction. Springer-Verlag, Bremen, Germany.

Hwang, I. S. Y. (2006). When does stacking become vertical sprawl? In The sustainable city IV: urban regeneration and sustainability (Vol. 93, pp. 283-292). WIT Press.

Keeling, D. J. (2008). Transportation geography - new regional mobilities. Progress in Human Geography, 32 (2), 275-283.

Kim, H., Jun, C., Cho, Y., \& Kim, G. (2008). Indoor Spatial Analysis using Space Syntax. In C. Jun (Ed.), ISPRS Silk Road for Information from Imagery (pp. 1065-1069), Beijing, China.

Kratchman, J. A. (2006). An Investigation on the Effects of Firefighter Counterflow and Human Behavior in a Six-Story Building Evacuation. University of Maryland, College Park, MD.

Kuligowski, E. D. (2008). Modeling human behavior during building fires. In (pp. 21). National Institute of Standards and Technology, Gaithersburg, MD.

Kwan, M.-P. (1999). Gender and Individual Access to Urban Opportunities: A Study Using Space-Time Measures. The Professional Geographer, 51 (2), 211-227.

Kwan, M.-P., \& Weber, J. (2003). Individual Accessibility Revisited: Implications for Geographical Analysis in the Twenty-first Century. Geographical Analysis, 35 (4), 341-353.

Kwan, M.-P., \& Weber, J. (2008). Scale and Accessibility: Implications for the Analysis of Land Use-Travel Interaction. Applied Geography, 28 (2), 110-123.

Le Site Officiel de l'Indice Passe-Partout (IPP). In http://www.ipp-online.org/ (2012).

Lee, J. (2004). A Spatial Access-Oriented Implementation of a 3-D GIS Topological Data Model for Urban Entities. Geoinformatica, 8 (3), 237-264.

Li, K.-J. (2008). Indoor Space: A New Notion of Space. In M. Bertolotto, C. Ray \& X. Li (Eds.), Web and Wireless Geographical Information Systems (Vol. 5373, pp. 1-3). Springer Berlin / Heidelberg.

Neutens, T., Delafontaine, M., Scott, D. M., \& De Maeyer, P. (2012). A GIS-based method to identify spatiotemporal gaps in public service delivery. Applied Geography, 32 (2), 253-264.

Neutens, T., Schwanen, T., Witlox, F., \& De Maeyer, P. (2008). My Space or Your Space? Towards a Measure of Joint Accessibility. Computers, Environment and Urban Systems, 32 (5), 331-342. 
Neutens, T., Versichele, M., \& Schwanen, T. (2010). Arranging place and time: A GIS toolkit to assess person-based accessibility of urban opportunities. Applied Geography, 30 (4), 561-575.

Otmani, R., Moussaoui, A., \& Pruski, A. (2009). A New Approach to Indoor Accessibility. International Journal of Smart Home, 3 (4), 1-14.

Predtechenskii, V. M., \& Milinskii, A. I. (1978). Planning for Foot Traffic Flow in Buildings. Amerind Publishing Co., New Delhi, India.

Sakkas, N., \& Pérez, J. (2006). Elaborating Metrics for the Accessibility of Buildings. Computers, Environment and Urban Systems, 30 (5), 661-685.

Santos, G., \& Aguirre, B. E. (2004). A Critical Review of Emergency Evacuation Simulation Models. In R. D. Peacock \& E. D. Kuligowsk (Eds.), NIST Workshop on Building Occupant Movement during Fire Emergencies (pp. 25-50), Gaithersburg, USA.

Thill, J.-C., Dao, T. H. D., \& Zhou, Y. (2011). Traveling in the Three-Dimensional City: Applications in Route Planning, Accessibility Assessment, Location Analysis and Beyond. Journal of Transport Geography, 19 (3), 405-421.

VanLandegen, L. D., \& Chen, X. (2012). Microsimulation of large-scale evacuations utilizing metrorail transit. Applied Geography, 32 (2), 787-797.

Versichele, M., Neutens, T., Delafontaine, M., \& Van de Weghe, N. (2012). The use of Bluetooth for analysing spatiotemporal dynamics of human movement at mass events: A case study of the Ghent Festivities. Applied Geography, 32 (2), 208-220.

Walton, L. A., \& Worboys, M. (2009). Indoor Spatial Theory. In www.spatial.maine.edu/ISAmodel/documents/IST_ISA09.pdf (pp. 6).

Wilson, M. (2010). Vertical Landscraping, a Big Regionalism for Dubai. International Journal of Urban and Regional Research, 34 (4), 925-940.

Yuan, J. P., Fang, Z., Wang, Y. C., Lo, S. M., \& Wang, P. (2009). Integrated network approach of evacuation simulation for large complex buildings. Fire Safety Journal, 44 (2), 266-275.

Zheng, X., Zhong, T., \& Liu, M. (2009). Modeling crowd evacuation of a building based on seven methodological approaches. Building and Environment, 44 (3), 437-445. 